\title{
Can polychlorinated biphenyl (PCB) signatures and enantiomer fractions be used for source identification and to age date occupational exposure?
}

\author{
David Megson ${ }^{\mathrm{a}, *}$, Jean-Françios Focant ${ }^{\mathrm{b}}$, Donald G. Patterson ${ }^{\mathrm{c}}$, Matthew Robson ${ }^{\mathrm{d}, \mathrm{e}}$, Maeve C. Lohan ${ }^{\mathrm{a}}$, \\ Paul J. Worsfold ${ }^{a}$, Sean Comber ${ }^{a}$, Robert Kalin ${ }^{f}$, Eric Reiner ${ }^{\mathrm{e}}$, Gwen O'Sullivan ${ }^{\mathrm{g}}$ \\ a Biogeochemistry Research Centre, SoGEES, Plymouth University, Plymouth, Devon PL4 8AA, UK \\ b Department of Chemistry, University of Liège, Allèe de la Chimie, Liège, Belgium \\ c Exponent Inc., One Capital City Plaza, Suite 1620, 3350 Peachtree Road, Atlanta, GA 30326, USA \\ d Department of Chemistry, Brock University, 500 Glenridge Ave, St. Catharines, ON L2S 3A1, Canada \\ e Ontario Ministry of the Environment and Climate Change, 125 Resources Road, Toronto, Ontario M9P 3V6, Canada \\ ${ }^{\mathrm{f}}$ Department of Civil and Environmental Engineering, University of Strathclyde, Glasgow G1 1XQ UK \\ g Department of Environmental Science, Mount Royal University, 4825 Mount Royal Gate SW, Calgary, Alberta T3E 6K6, Canada
}

\section{A R T I C L E I N F O}

\section{Article history:}

Received 12 February 2015

Received in revised form 14 April 2015

Accepted 14 April 2015

Available online $\mathrm{xxxx}$

\section{Keywords:}

Polychlorinated biphenyls (PCBs)

Human exposure

GC $\times$ GC-TOFMS

Chemical fingerprinting

PCB atropisomers

Chiral

Enantiomer fractions

\begin{abstract}
A B S T R A C T
Detailed polychlorinated biphenyl (PCB) signatures and chiral Enantiomer Fractions (EFs) of CB-95, CB-136 and CB-149 were measured for 30 workers at a transformer dismantling plant. This was undertaken to identify sources of exposure and investigate changes to the PCB signature and EFs over different exposure periods. Approximately $1.5 \mathrm{~g}$ of serum was extracted and $\mathrm{PCB}$ signatures were created through analysis by comprehensive two-dimensional gas chromatography with time-of-flight mass spectrometry (GC $\times$ GC-TOFMS) and EFs calculated following analysis by gas chromatography with high resolution mass spectrometry (GC-HRMS). A total of 84 PCBs were identified in the serum samples with concentrations of the 7 indicator PCBs ranging from $11-350 \mathrm{ng} \mathrm{g}^{-1}$ of serum (1.2-39 $\mu \mathrm{g} \mathrm{g}^{-1}$ lipid). The PCB signatures were interpreted using principal component analysis (PCA) which was able to distinguish workers with background or recent minimal exposure from those with prolonged occupational exposure. Occupationally exposed individuals had a similar PCB profile to Aroclor A1260. However, individuals with prolonged exposure had depleted proportions of several PCB congeners that are susceptible to metabolism (CB-95, CB-101 and CB-151) and elevated proportions of PCBs that are resistant to metabolism (CB-74, CB-153, CB-138 and CB-180). The results also identified a third group of workers with elevated proportions of CB-28, CB-60, CB-66, CB-74, CB-105 and CB-118 who appeared to have been exposed to an additional source of PCBs. The results show near complete removal of the CB-95 E2 enantiomer in some samples, indicating that bioselective metabolism or preferential excretion of one enantiomer occurs in humans. By considering PCB concentrations along with detailed congener specific signatures it was possible to identify different exposure sources, and gain an insight into both the magnitude and duration of exposure.
\end{abstract}

(c) 2015 Elsevier Ltd. All rights reserved.

\section{Introduction}

Polychlorinated biphenyls (PCBs) are a group of 209 chlorinated organic compounds that were widely used throughout the 20th century. Whilst PCBs have been largely phased out of commercial/industrial use, they remain an important legacy contaminant (O'Sullivan and Sandau, 2013). They are highly persistent and can still be found in closed systems in some countries, e.g. as dielectric fluids in electrical equipment and transformers. Many of these transformers containing

\footnotetext{
* Corresponding author at: Biogeochemistry Research Centre, Plymouth University, Plymouth, Devon PL4 8AA, UK.

E-mail address: dpmegson@hotmail.co.uk (D. Megson).
}

PCBs are in the process of being replaced, and this process therefore presents the potential of PCB exposure for humans working in dismantling plants. In these instances it is important not only to determine the extent of exposure, along with the potential risks to human health, but to also establish the source and age date the exposure.

As PCBs were produced as commercial mixtures, such as Aroclors, each blend has a specific congener profile (signature) based on the relative proportions of each PCB in the total mixture. This signature can be used to easily distinguish commercial mixtures, however environmental investigations involving humans are more complex as there are often multiple potential sources of PCB exposure. The signature can be altered by changes such as volatilization, dissolution and biodegradation (Jaspers et al., 2013; Johnson et al., 2006). The signature in humans can also vary depending on different exposure pathways e.g. 
oral, inhalation or dermal, and can be altered through post-uptake processes such as biotransformation and elimination (Jaspers et al., 2013; Megson et al., 2013a). If a dominant source of exposure can be identified then alterations to this signature from post-uptake processes such as biotransformation and elimination may provide useful information to distinguish between recent and prolonged exposure. The sera of an individual who has been historically exposed may contain higher proportions of the PCBs that are more resistant to biotransformation and elimination. Due to all of the subtle changes that can occur to the PCB profile, it is imperative that signatures are created using detailed congener specific datasets when attempting to identify the source of exposure. Analysis using comprehensive two dimensional chromatography has proven to be an excellent technique for this purpose as it is able to separate over 190 individual PCB congeners (Focant et al., 2004; Korytar et al., 2006; Harju et al., 2003; Zapadlo et al., 2011; Megson et al., 2013b).

Of the 209 PCBs there are 19 which are predicted to exist as stable atropisomers (Oki, 1983). They have a high degree of ortho chlorine substitution which inhibits rotation, and asymmetrical meta and para substitution on each biphenyl, resulting in two optical isomers. In commercial mixtures both enantiomers are produced in equal proportions; however in animals, metabolic processes such as enzyme mediated oxidation have been proven to preferentially target one stereoisomer, resulting in atropisomeric enrichment (Harrad et al., 2006; Wong et al., 2002; Wu et al., 2014). Therefore, the sera of an individual who has recently been exposed to a commercial PCB mixture may be expected to contain near equal proportions of each stereoisomer, whereas the sera of a historically exposed individual may show a greater degree of atropisomeric enrichment. However, this signal is likely to be complicated by interferences such as other background sources of PCBs.

The goal of this study was to determine if PCB signatures and enantiomer fractions could be used to identify the exposure source and distinguish between recent and prolonged exposure periods for 30 workers at a transformer dismantling plant.

\section{Experimental}

\subsection{Sample collection}

Samples of whole blood were collected from 30 people working at a transformer dismantling plant. Samples were obtained from workers performing a range of different roles at the plant, including workers on the dismantling floor who were likely to have had direct contact with PCBs and those who were not expected to have had any direct contact with PCBs due to their job roles. Samples were obtained from employees who had been working at the plant from 3-21 years. However, information on occupation and length of time at the plant was not available for three participants (id no. S028, S029 and S030). A $10 \mathrm{~mL}$ sample of whole blood was obtained from each worker, the blood was collected in vacutainers, then centrifuged and the serum collected and stored at $-20{ }^{\circ} \mathrm{C}$ until analyses, following a reported procedure (Focant et al., 2006). All samples were anonymized using a double blind coding system between time of sampling and time of analyses to ensure protection of individual privacy.

Total lipid concentrations were determined by enzymatic analysis which was performed by a sub-contractor clinical laboratory on a dedicated $2 \mathrm{~mL}$ serum sub-sample. Four types of lipids were targeted and measured; triglycerides, total cholesterol, non-esterified (free) cholesterol, and phospholipids. Sample sizes were as follows: triglycerides $(2 \mu \mathrm{L})$, total cholesterol $(2 \mu \mathrm{L})$, non-esterified (free) cholesterol $(50 \mu \mathrm{L})$, and phospholipids $(20 \mu \mathrm{L})$. Total lipid concentrations were estimated using the summation method of Akins et al. (1989). The total lipid content was expressed in $\mathrm{g} \mathrm{L}^{-1}$. For the inter-conversion of volumetric and gravimetric data, a value of $1.026 \mathrm{~g} \mathrm{~mL}^{-1}$ for serum specific gravity was used.

\subsection{Sample preparation}

All reagents required for extraction and clean-up were sourced specifically for dioxin, furan and PCB analysis or of the closest grade available of similar quality. Approximately $1.5 \mathrm{~g}$ of serum was accurately weighed (to 4 decimal places) and transferred to a vial and $5 \mu \mathrm{L}$ of ${ }^{13} \mathrm{C}_{12}$ labelled CB-60, CB-127 and CB-159 at a concentration of $100 \mathrm{pg} \mu \mathrm{L}^{-1}$ (CIL-EC-5370 EN-1948-4 PCB sampling standard) was added to determine recovery. A volume of formic acid equal to the mass of the sample was added to the serum followed by the same volume of high purity water. During each addition the solution was vortexed and allowed to degas for several minutes. The whole extract was then applied to a $\mathrm{C}_{18}$ SPE cartridge $(1 \mathrm{~g} / 6 \mathrm{~mL})$ and the PCBs were eluted with hexane. The eluent was treated using EPA method 3665A sulphuric acid/permanganate clean-up followed by EPA method 3620 Florisil clean-up. Extracts were reduced to approximately $50 \mu \mathrm{L}$ by nitrogen evaporation and $100 \mu \mathrm{L}$ of ${ }^{13} \mathrm{C}_{12}$ labelled PCBs (CIL-EC-5367 CDC PCB Spiking Standard), each at a concentration of $7.5 \mathrm{pg} \mu \mathrm{L}^{-1}$, were added. Extracts were left overnight to evaporate to incipient dryness (the spiking standard contained a dodecane keeper). Samples were reconstituted with $10 \mu \mathrm{L}$ of hexane prior to analysis.

\subsection{Congener specific analysis by GC $\times$ GC-TOFMS}

Analysis was conducted based on the method described by Megson et al. (2013b) which is summarised below. Samples were analysed using a time-of-flight mass spectrometer (LECO, St. Joseph, MI Pegasus 4D) coupled to a two dimensional gas chromatograph (Agilent Technologies 7890A) equipped with a thermal modulator (LECO, St. Joseph, MI). The gas chromatograph was fitted with an Rtx-PCB $(60 \mathrm{~m} \times$ $0.18 \mathrm{~mm} \times 0.18 \mu \mathrm{m}){ }^{1} \mathrm{D}$ column and an Rxi-17 Sil MS $(1.5 \mathrm{~m} \times$ $0.18 \mathrm{~mm} \times 0.18 \mu \mathrm{m})^{2} \mathrm{D}$ column. One microlitre of sample was injected in splitless mode.

Procedural blanks were prepared for each batch of 8 samples. Contamination with $\mathrm{CB}-11$ was identified in the blanks and so this congener was excluded from the results. All samples were spiked with two sets of ${ }^{13} \mathrm{C}_{12}$ labelled internal standards which were used to quantify PCB concentrations and calculate recovery. Quantification was undertaken through isotope dilution; calibration data was produced for 42 of the most commonly encountered congeners (CIL-EC-4133 DSJ PCB Mixture). Quantification of other congeners present in the samples was undertaken using the calibration data from the closest eluting calibrated congener with the same level of chlorination. Recovery for all samples was within the accepted range specified by EPA method $1668 \mathrm{C}$ ( $10 \%$ to $145 \%)$, the mean recovery was $55 \%$ ( $\pm 16 \%$; $1 \sigma)$. Concentrations were recovery corrected and lipid normalised and reported as $\mathrm{ng} \mathrm{g}^{-1}$ lipid weight. The instrument limit of detection (LOD) was estimated empirically using the calibration standard mixtures, LODs for CB-18 and CB-206 were calculated at a concentration of at 1 and $50 \mathrm{pg} \mu \mathrm{L}^{-1}$ respectively (LODs for the other 40 PCBs are presented in Supplementary Information 2). Accuracy and precision were measured for the sum of the European Union 7 indicator congeners (EC7; CB-28, CB-52, CB-101, CB-118, CB-138, CB-153, CB-180) by analysing a $10 \mathrm{ng} \mu \mathrm{L}^{-1}$ Aroclor 1248 standard three times. The accuracy of the sum of the EC7 congeners for the three samples was $105 \%$ ( $\pm 0.9 \% ; 1 \sigma)$.

\subsection{Chiral analysis by GC-HRMS}

The Enantiomeric Fractions (EFs) of CBs 95, 136 and 149 were analysed according to the method of Robson and Harrad (2004), using an Agilent 7890 gas chromatograph coupled to a Micromass AutoSpec Premier high resolution mass spectrometer (GC-HRMS) tuned to greater than 10000 mass resolution. The two most abundant isotopes of each enantiomer were recorded in Selected Ion Recording Mode (SIR). This was $m / z 325.88040$ and 327.87750 for CB 95 and $m / z 359.84150$ and 361.83850 for CBs 136 and 149. These PCBs were chosen because they 
are; (a) able to be baseline separated on the Chirasil-Dex column, (b) free from any co-eluting congeners that may bias the results and (c) normally present in the environment in high enough concentrations to be accurately measured.

The chromatographic performance of the method was assessed prior to each run of 8 samples by analysing a 1:1:1 mixture of Aroclors 1248, 1252 and 1260.

Enantiomeric Fractions were calculated as per Harner et al. (2000). Whereby

$\mathrm{EF}=\mathrm{E} 1 /(\mathrm{E} 1+\mathrm{E} 2)$

where E1 equals the first eluting or the $(+)$ enantiomer and E2 the second eluting enantiomer.

Samples where only accepted for quantitation if the enantiomeric fractions of the three atropisomers studied were $0.50( \pm 0.01)$ in the Aroclor mixture, the least abundant enantiomer of the pair had a signal to noise $(\mathrm{S}: \mathrm{N})$ ratio greater than $10: 1$, the isotope ratios were within $20 \%$ of their theoretical values and the analytical recovery of the sample was greater than $30 \%$. The instrument LOD was estimated by analysing a standard mixture of CB-95 and CB-149, LODs were calculated at a concentration of $2.5 \mathrm{pg} \mathrm{L}^{-1}$ per enantiomer. Procedural blanks were prepared for each batch of 8 samples; no chiral PCBs were detected in the blanks above the limits of detection.

\section{Results and discussion}

\subsection{Concentrations of PCBs in workers at a transformer dismantling plant}

A total of 84 different PCB congeners were identified in the serum of the 30 workers at the transformer dismantling plant. Concentrations of the 7 indicator PCBs ranged from $11-350 \mathrm{ng} \mathrm{g}^{-1}$ of serum (1.2$39 \mu \mathrm{g} \mathrm{g}^{-1}$ lipid). Background concentrations of these congeners in humans are in the range of $0.1-10 \mathrm{ng} \mathrm{g}^{-1}$ serum $\left(0.01-1 \mu \mathrm{g} \mathrm{g}^{-1}\right.$ lipid) (Longnecker, 2001). This shows that some workers had close to background exposure whereas others had elevated PCB concentrations indicating that they may have been exposed to $\mathrm{PCBs}$ through their occupation. The PCB concentrations in the different workers are summarised in Table 1. The results show that the workers with job roles involving direct contact with $\mathrm{PCB}$ generally had higher $\mathrm{PCB}$ concentrations than those with no direct contact. The mean concentration of the EC7 PCBs in workers with direct contact was $17.2 \mu \mathrm{g} \mathrm{g}^{-1}$ lipid $( \pm 13.0 ; 1 \sigma)$ which was significantly greater (P-value 0.004$)$ than workers with no direct contact $\left(5.83 \mu \mathrm{g} \mathrm{g}^{-1}\right.$ lipid $\left.( \pm 6.90 ; 1 \sigma)\right)$. However, this was not true for all workers, as a concentration of $21.0 \mu \mathrm{g} \mathrm{g}^{-1}$ lipid was recorded in one worker who was believed to have had no direct contact with PCBs. PCB concentrations generally increased with the number of years the employee had worked with the company (Fig. 1A), although this increase was not statistically significant (P-value of 0.052 ). However, when PCB concentrations of the 18 workers who were working at the dismantling plant with direct contact dismantling transformers were considered, this increase was statistically significant (P-value of 0.000) (Fig. 1B). Regression was also undertaken using the MannKendal test for discrete data which again showed a significant increase (P-value of 0.001). Although the PCB concentration is well correlated with the number of years an individual has worked at the company it should not be used in isolation as proof that occupational exposure has occurred. Higher PCB concentrations have been reported in older individuals as a result of accumulation of the more persistent congeners and exposure to higher historical background concentrations (Megson et al., 2013a; Quinn and Wania, 2012). There are also several physiological characteristics, such as body fat, serum albumin and age, that can

Table 1

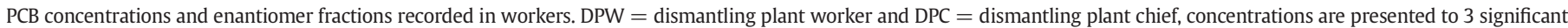
figures and EFs to 2 decimal places. $<$ LOD = below the limit of detection (i.e. S:N ratio $<10$ ).

\begin{tabular}{|c|c|c|c|c|c|c|c|c|c|c|c|}
\hline & \multirow[t]{2}{*}{ Sample ID } & \multirow[t]{2}{*}{$\begin{array}{l}\text { Years with the } \\
\text { company }\end{array}$} & \multirow[t]{2}{*}{$\begin{array}{l}\text { Concentration in } \\
\text { serum } \\
\left(\mathrm{ng} \mathrm{g}^{-1}\right)\end{array}$} & \multicolumn{2}{|c|}{$\begin{array}{l}\text { Lipid corrected } \\
\text { concentration in serum } \\
\left(\mu \mathrm{g} \mathrm{g}^{-1} \text { lipid }\right)\end{array}$} & \multicolumn{6}{|c|}{ Enantiomer fractions } \\
\hline & & & & $\begin{array}{l}\text { Per } \\
\text { sample }\end{array}$ & Mean $[ \pm 1 \sigma]$ & CB-95 & Mean $[ \pm 1 \sigma]$ & CB-136 & Mean $[ \pm 1 \sigma]$ & CB-149 & Mean $[ \pm 1 \sigma]$ \\
\hline \multirow[t]{8}{*}{ No direct contact } & S001 & $19-21$ & 18.4 & 2.49 & 5.83 & 0.51 & 0.63 & $<\mathrm{LOD}$ & 0.44 & 0.47 & 0.37 \\
\hline & S002 & $19-21$ & 13.7 & 1.49 & {$[ \pm 6.90]$} & 0.74 & {$[ \pm 0.14]$} & $<$ LOD & {$[ \pm 0.00]$} & $<$ LOD & {$[ \pm 0.08]$} \\
\hline & S003 & $10-12$ & 11.2 & 1.23 & & 0.41 & & $<\mathrm{LOD}$ & & 0.41 & \\
\hline & S004 & $4-6$ & 29.4 & 4.08 & & 0.55 & & $<$ LOD & & 0.26 & \\
\hline & S005 & $19-21$ & 166 & 21 & & 0.59 & & $<$ LOD & & 0.31 & \\
\hline & S006 & $19-21$ & 86.3 & 11.1 & & 0.66 & & 0.45 & & 0.39 & \\
\hline & S007 & $19-21$ & 26.6 & 1.93 & & 0.78 & & 0.44 & & 0.41 & \\
\hline & S008 & $4-6$ & 21 & 3.32 & & 0.82 & & $<\mathrm{LOD}$ & & $<$ LOD & \\
\hline \multirow[t]{19}{*}{ Direct contact } & S009 & $10-12$ & 28.4 & 3.76 & 17.2 & 0.59 & 0.74 & 0.45 & 0.47 & 0.47 & 0.36 \\
\hline & S010 & $13-15$ & 62.8 & 9.41 & {$[ \pm 13.0]$} & 0.51 & {$[ \pm 0.11]$} & $<$ LOD & {$[ \pm 0.05]$} & 0.48 & {$[ \pm 0.07]$} \\
\hline & S011 & $4-6$ & 73.1 & 6.53 & & 0.88 & & 0.51 & & 0.37 & \\
\hline & S012 & $7-9$ & 70.1 & 13.2 & & 0.88 & & $<$ LOD & & 0.29 & \\
\hline & S013 & $0-3$ & 27.7 & 3.37 & & 0.87 & & 0.57 & & 0.44 & \\
\hline & S014 & $10-12$ & 246 & 36.2 & & 0.87 & & 0.43 & & 0.21 & \\
\hline & S015 & $13-15$ & 163 & 20.6 & & 0.82 & & $<\mathrm{LOD}$ & & $<\mathrm{LOD}$ & \\
\hline & S016 & $7-9$ & 138 & 14.4 & & 0.79 & & 0.47 & & 0.32 & \\
\hline & S017 & $16-18$ & 121 & 19.4 & & 0.72 & & 0.43 & & 0.30 & \\
\hline & S018 & $16-18$ & 353 & 38.5 & & 0.66 & & 0.46 & & 0.35 & \\
\hline & S019 & $4-6$ & 16.8 & 3 & & 0.60 & & $<\mathrm{LOD}$ & & 0.33 & \\
\hline & S020 & 19-21 & 300 & 37.7 & & 0.87 & & $<$ LOD & & $<$ LOD & \\
\hline & S021 & $7-9$ & 95.2 & 14.2 & & 0.74 & & $<\mathrm{LOD}$ & & 0.37 & \\
\hline & S022 & $19-21$ & 231 & 39.3 & & 0.67 & & 0.47 & & 0.35 & \\
\hline & S023 & $13-15$ & 62.5 & 10.4 & & 0.67 & & 0.43 & & 0.35 & \\
\hline & S024 & $4-6$ & 37.5 & 4.16 & & 0.82 & & 0.51 & & 0.32 & \\
\hline & S025 & 4-6 & 23.4 & 4.05 & & 0.66 & & $<\mathrm{LOD}$ & & 0.44 & \\
\hline & S026 & $7-9$ & 134 & 27.3 & & 0.66 & & $<$ LOD & & 0.44 & \\
\hline & S027 & $13-15$ & 149 & 21.2 & & 0.77 & & $<\mathrm{LOD}$ & & $<$ LOD & \\
\hline \multirow[t]{3}{*}{ Unknown } & S028 & Unknown & 26.1 & 3.55 & 13.7 & 0.82 & 0.78 & 0.52 & 0.55 & 0.36 & 0.35 \\
\hline & S029 & Unknown & 187 & 30.4 & {$[ \pm 14.6]$} & 0.91 & {$[ \pm 0.15]$} & 0.59 & {$[ \pm 0.048]$} & 0.27 & {$[ \pm 0.08]$} \\
\hline & S030 & Unknown & 50.7 & 7.14 & & 0.61 & & $<$ LOD & & 0.43 & \\
\hline
\end{tabular}



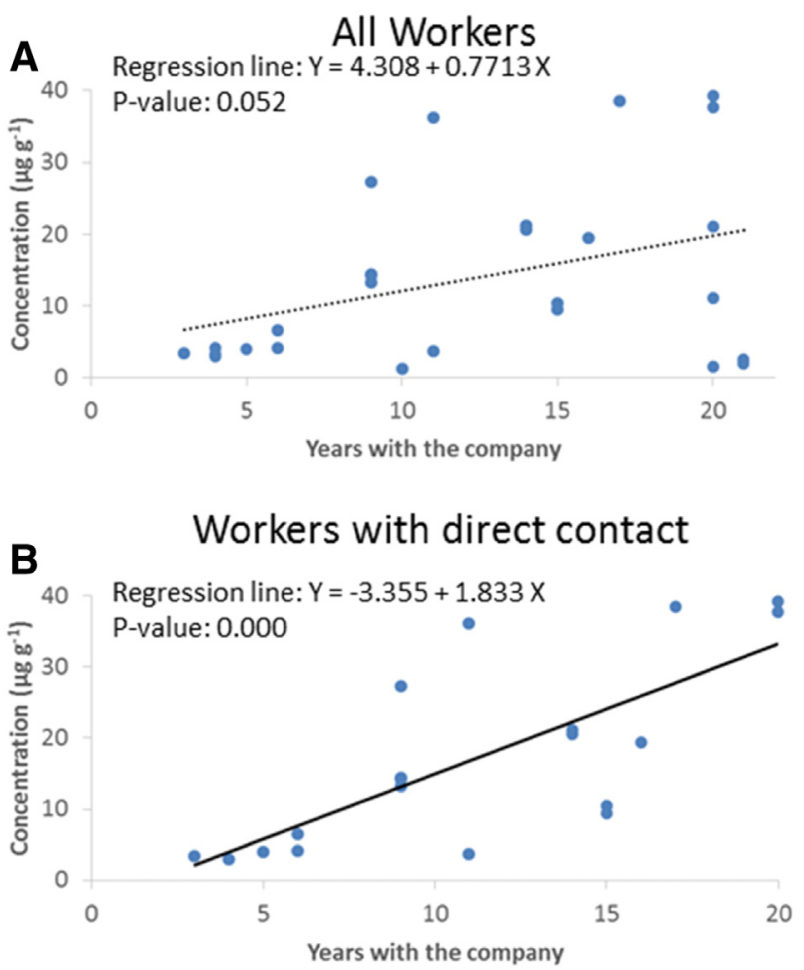

Fig. 1. Relationship between $\mathrm{PCB}$ concentration and number of years a worker had been with the company. Results are presented for all workers (Fig. 1A) and only those with direct contact dismantling transformers (Fig. 1B).

influence the uptake and retention of PCBs along with social preferences such as diet and smoking (Axelrad et al., 2009; Brown and Lawton, 2001; Jain and Wang, 2011; Weintraub and Birnbaum, 2008). This highlights the importance of looking at the specific PCB signature of each individual to determine their exposure source rather than relying solely on a total PCB concentration.

\subsection{Statistical evaluation of PCB signatures}

Fifty four PCBs were consistently detected in $>60 \%$ of the samples. These were quantified, percent normalised and presented as bar charts to show the PCB signature in each participant (Supplementary Information 1). Only three different Aroclor blends were understood to have been used in transformers; these were A1242, A1254 and A1260 (Johnson et al., 2006). As the dismantling plant was in Europe, transformers were likely to have contained a variety manufacturers products from the European market, including Aroclors, Phenoclors, Pyralenes and Clophens, however there is a very high degree of similarity in the signature from blends with an equivalent chlorine content (Johnson et al., 2006). The signature for the majority of workers was visually similar to the signature of A1260 (Supplementary Information 1) which provides further evidence to suggest that occupational exposure had occurred. However, further assessment was undertaken to confirm if this was the source of exposure or if other potential sources were important.

The PCB signatures of the workers were assessed using principal component analysis (PCA). Where a PCB was not detected it was included in the dataset as a ' 0 '. As part of the data quality check, other values such as LOD/2 were substituted for ' 0 ', but these had no observable effect on the data output and so the '0's were retained. To reduce any bias from a high proportion of non-detects for a specific congener, PCBs that were not detected in over $60 \%$ of samples (i.e. PCBs present in less than 18 out of the 30 samples) were removed from the analysis following the guidance of Helsel (2006). This resulted in a dataset of 54 PCBs in 30 participants. Before performing PCA the data were normalised by transformation to a percent metric to remove concentration/dilution effects. The data were then mean centred and scaled using a Z-transform (autoscale transform) to prevent high concentration variables from dominating the analysis (Johnson et al., 2007). Principal component 1 accounted for $33.1 \%$ of the variation and principal component 2 accounted for a further $18.2 \%$. The scores plot is presented as Fig. 2 and the loadings plot as Fig. 3.

The scores plot (Fig. 2) displayed a three end member system, showing that there were three groups of workers, each with a different PCB signature. Two of the three groups were linked to the duration that the participant had been working at the plant and their job, i.e. involving either direct contact with PCBs or only indirect contact. One group may therefore represent participants with prolonged occupational exposure and the other group exposure to background levels or a recent minimal exposure. However, the third group was comprised of participants who all had different ages, jobs and years at the company and may therefore be linked to an additional source(s) of exposure. Bar charts were produced to identify differences in the PCB signature between the three groups and aid the source identification process. Fig. 4 displays the signature of A1260 along with the signatures of three participants (selected using the PCA scores plot) to represent the three groups. These were; participant number S020 who had worked at the plant for 19-21 years (representing a prolonged exposure to A1260), participant number S013 who had been at the company for 0-3 years (representing a recent minimal exposure to A1260), and participant number S021 (representing a suspected additional exposure source).

\subsection{Source identification and age dating exposure}

\subsubsection{Occupational exposure to A1260}

Participants with a negative score on PC1 and PC2 were comprised of workers who had been working at the dismantling plant for more than 10 years, with direct contact through dismantling transformers. The signatures from these samples is visually similar to the profile of A1260 but with depleted proportions of several of the less chlorinated biphenyls, CB-88 \& 95, CB-90 \& 101 and CB-151 (Fig. 4). These are congeners predominantly containing a phenyl group with un-chlorinated meta and para positions (i.e. 2,5-chlorine substitution), which are particularly susceptible to metabolic attack by P450 cytochromes (Letcher et al., 1999). The signature also contained elevated proportions of $\mathrm{CB}-74$, CB-153, CB-138 and CB-180 (Fig. 4). These are congeners containing a phenyl group with 2,4,5-substitution which are particularly resistant to biotransformation and elimination (Megson et al., 2013a). Interestingly this group also included several participants with jobs not believed to involve direct exposure, although they had low PCB concentrations their signature was similar to A1260. The signature shows that these individuals appear to have been exposed to PCBs through occupational exposure, although the total PCB concentrations indicate exposure was only minimal.

\subsubsection{Background or recent minimal occupational exposure}

Participants with a positive score on PC2 comprised of workers who had been working at the plant for a relatively short period of time ( $<6$ years), along with those with jobs that did not involve direct exposure. All of these individuals contained significantly lower (P-value 0.000 ) EC7 PCB concentrations (mean value of $3.2 \mu \mathrm{g} \mathrm{g}^{-1}$ lipid $( \pm 1.0 ; 1 \sigma)$ ) than the rest of the samples (mean value of

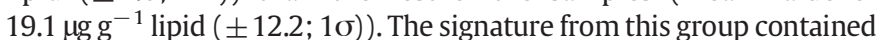
higher proportions of several episodic congeners such as $C B-8, C B-18$, CB-31, CB-52 and CB-151 (Fig. 3), indicating a recent exposure. The signature also displayed slightly elevated proportions of many of the lower chlorinated PCBs that were not present in high concentrations in A1260 such as CB-28, CB-74, CB-99 and CB-118 (Fig. 4), indicating a background exposure (Fig. 2). 


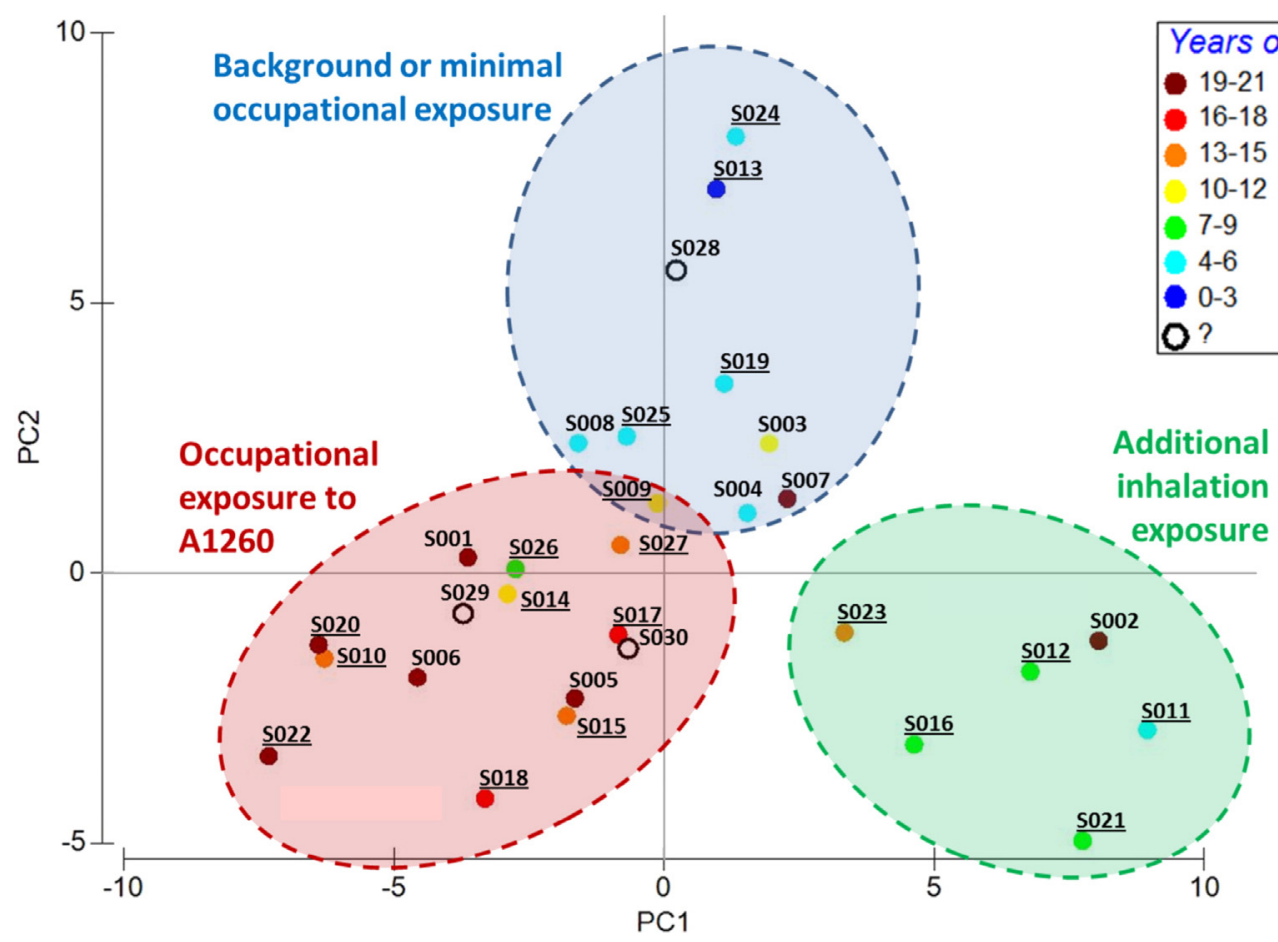

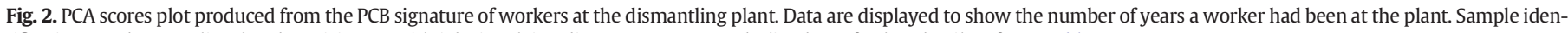
tification numbers are listed and participants with jobs involving direct contact are underlined. For further details refer to Table 1.

\subsubsection{Additional inhalation exposure}

In some instances inhalation has proven to be an important PCB exposure pathway (DeCaprio et al., 2005; Herrick et al., 2011). In a school in Boston (U.S.) inhalation of PCBs leaching from caulking materials and sealants was determined as the main route of exposure for teachers. This exposure resulted in the teachers having a distinctive PCB signature, with proportions of the less chlorinated $\mathrm{PCBs}$ such as $\mathrm{CB}-8$, CB-33, CB-37, CB-41, CB-47 and CB-136 up to five times higher than the control group (Herrick et al., 2011). In this current study, participants with a positive score on PC1 and negative score on PC2 had a signature similar to A1260, but with the addition of high proportions of several less chlorinated PCBs such as CB-28, CB-60, CB-66 and CB-74,

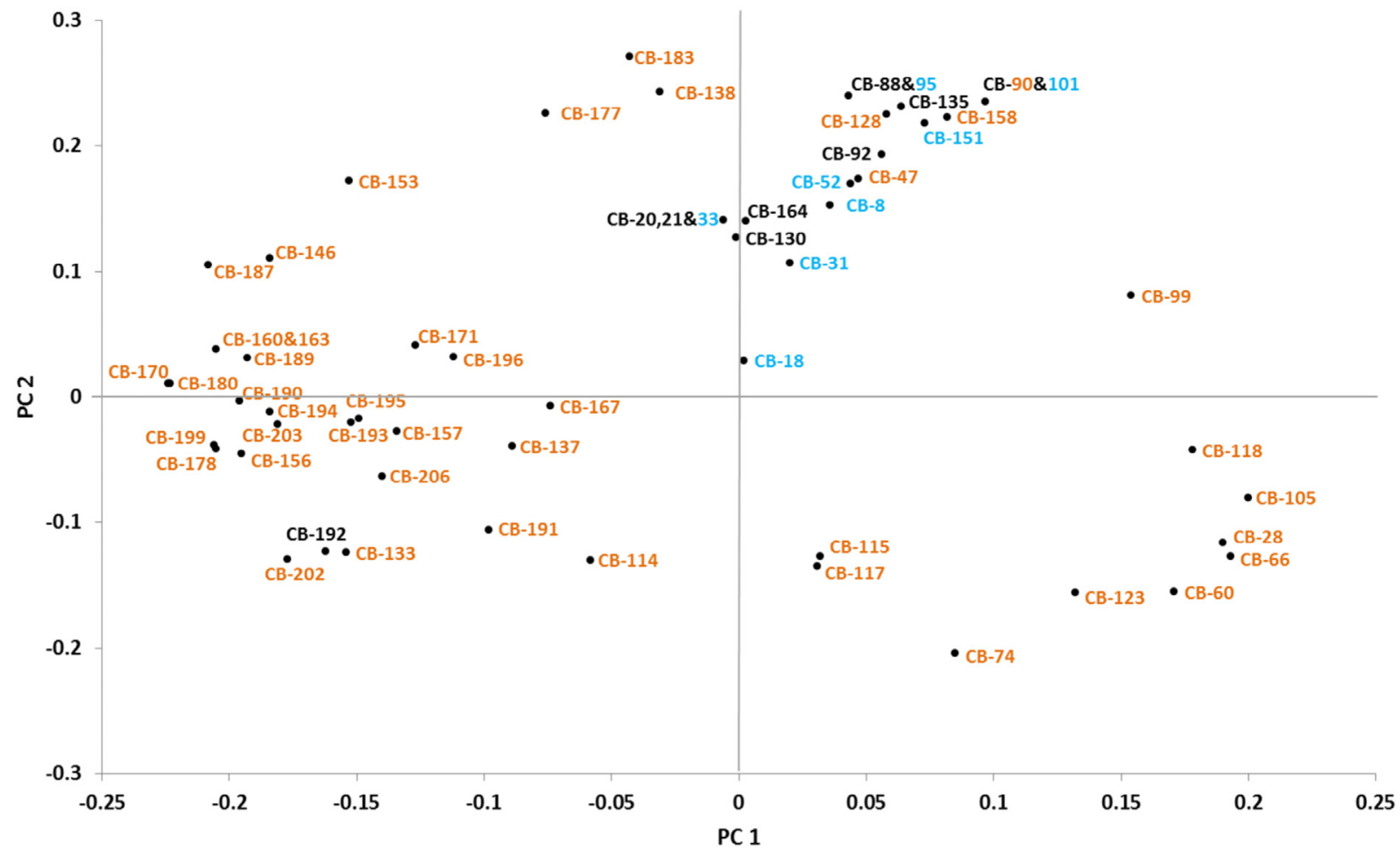

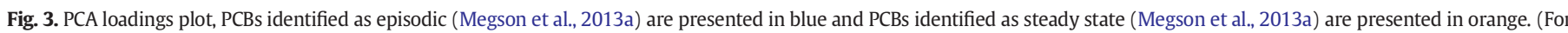
interpretation of the references to color in this figure legend, the reader is referred to the web version of this article.) 


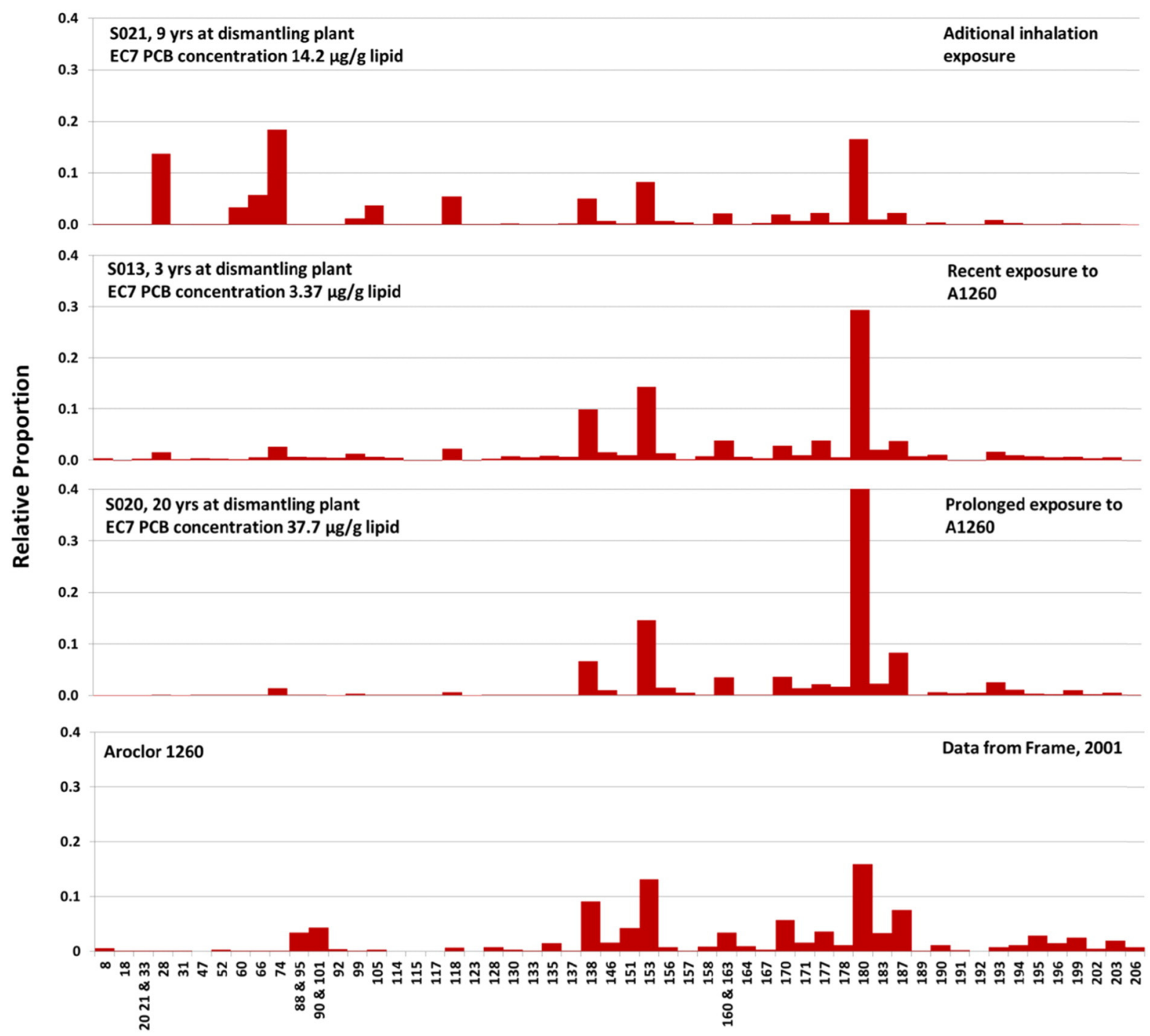

Fig. 4. PCB signature for the serum samples obtained from participants S020, S013 and S021 compared with the signature from A1260.

along with CB-105 and CB-118. This group was comprised of participants who all had different ages, jobs and years at the company. The signatures all contained higher proportions of more volatile PCBs which have been previously linked to indoor air sources such as leaching from sealants and caulking materials (Harrad et al., 2005; Herrick et al., 2011; Kohler et al., 2005). Several of the congeners present in higher proportions (CB-28, CB-60 and CB-66 and CB-74) have also been linked to exposure from capacitors in electrical equipment (Luotamo et al., 1993). Concentrations of PCBs in individuals from this group were similar to those from the group with prolonged exposure to A1260. Therefore, the results indicate that this group was exposed to an additional source of PCBs through inhalation, possibly originating from leaching of materials at the home rather than from their workplace (Fig. 2).

\subsubsection{Enantiomeric fractions}

To further elucidate the potential sources of exposure to these individuals we also examined the enantiomer signatures of thee chiral PCBs (CB-95, CB-136 and CB-149) in the samples to ascertain if there were any trends in enantio-specific processing of these congeners that could be related to variations in the exposure of the workers, as well as potentially providing further insight into the pharmacokinetics of these pollutants in humans. To our knowledge this is the first time that analysis of this type has been done for human serum samples despite the widespread use of this matrix in human bio-monitoring programmes (e.g. Canadian Health Measures Survey, United Sates Human Bio-monitoring Program).

The enantiomeric fractions of $\mathrm{CB}-95$ and $\mathrm{CB}-149$ recorded in the workers varied considerably ( 0.41 to 0.91 and 0.21 to 0.48 , respectively), whereas fractions of CB-136 remained close to racemic ( 0.43 to 0.59) (Table 1). For CB-95 sera from most participants contained higher proportions of the E1 or $(+)$ enantiomer than the E2 or $(-)$ enantiomer, indicating that significant enantioselective metabolism, elimination or sequestration of this enantiomer may have occurred. In one worker (S029) the proportion of E1 enantiomer was over 10 times greater than the E2 enantiomer. This mirrors the work of Chu et al. (2003) who found similar results for CB-95 in human liver samples. However in this current study the extent of change was much greater than was reported in Chu et al. (2003), with the results showing evidence of near complete removal of the $E 2$ enantiomer in some participants. For CB-149 sera from all participants contained higher proportions of E2. In one worker the proportion of E2 was over 3.5 times greater than E1 (S014).

No clear trends in enantiomeric signatures with the exposure type or duration of work were identified in this study (Fig. 5). However, there was a weak correlation between the EFs of CB-95 and CB-149 with EC7 PCB concentrations (Fig. 5). Participants with higher PCB concentrations tended to display a greater degree of enrichment of the E1 

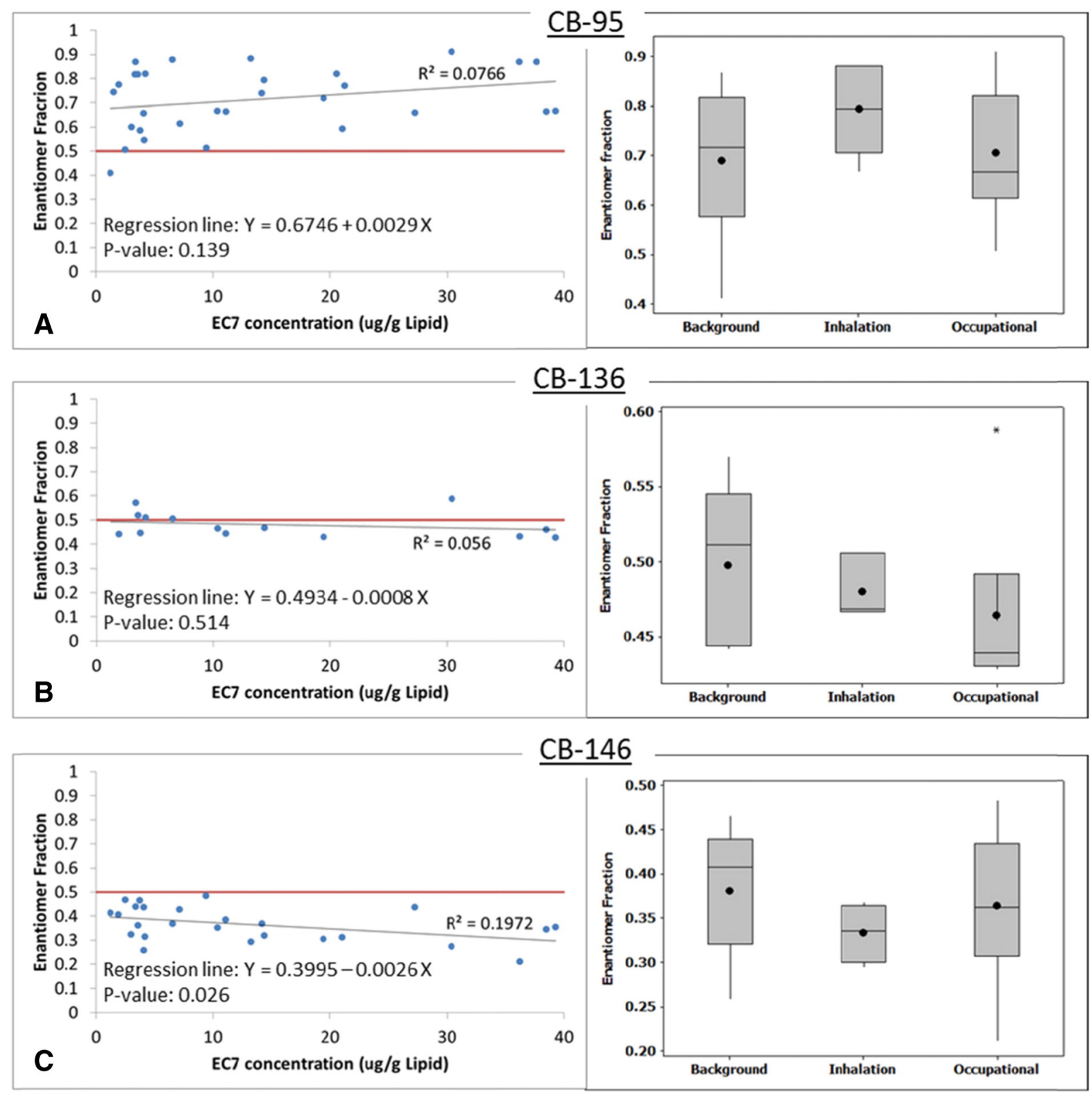

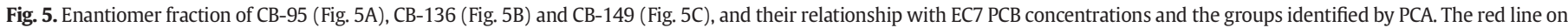
the scatter plots represents an EF of 0.5 (i.e. a racemic mixture). Box plots display the interquartile range, median and mean $(\bullet$ ).

enantiomer of CB-95 (P-value 0.139) and E2 enantiomer of CB-149 (P-value 0.026). This suggests that there may be a concentration dependent element to the metabolism of these congeners in humans. The high variability in the data for these two congeners also indicates that there is significant intra-individual variation in the enantiospecific processing of these contaminants.

Importantly these data also indicate that PCB profiles measured in sera may not fully match those found in other bodily tissues. The data recorded here are in contrast to those recorded by Chu et al. (2003) for CB-95, CB-132 and CB-149 in human muscle, kidney and brain samples that were all racemic or nearly racemic. However, they do match those recorded by Chu et al. (2003) for liver samples which were largely non-racemic in nature. This suggests that sera may in fact reflect liver profiles only, rather than whole body signatures. This has potentially important implications for sera based human biomonitoring programmes such as the Canadian Health Measures Survey (CHMS) and the National Health and Nutrition Examination Survey (NHANES) as it suggests that they may underestimate the true PCB burden and profiles of subjects. However, further work is needed to confirm this hypothesis. These data also suggest that the enantiomeric profile of the PCBs should be taken into account when assessing the toxicity of any potential exposure as the persistance and consequent effects of the enantiomers and their metabolic products may be significantly different (Kodavanti and Curras-Collazo, 2010). This is especially relevant for CB-95 which exhibits the highest potency and efficacy towards the ryanodine receptor which has been linked to developmental neurotoxicity (Schantz et al., 1997).

\section{Conclusions}

Identifying the source and age dating human exposure to $\mathrm{PCBs}$ is a highly complex task. This is due to the wide range of PCB sources that humans are exposed to along with different exposure pathways and processes such as volatilization, dissolution, biodegradation and postuptake processes that can all alter the original PCB signature. However, by considering PCB concentrations along with detailed congener 
specific signatures it was possible to identify different sources, and gain an insight into both the magnitude and duration of exposure. Occupationally exposed individuals had a similar PCB profile to Aroclor A1260. Individuals with prolonged exposure had depleted proportions of several PCB congeners that are susceptible to metabolism (CB-95, CB-101 and CB-151), and elevated proportions of $\mathrm{PCBs}$ that are resistant to metabolism (CB-74, CB-153, CB-138 and CB-180). A group of workers was also identified with a suspected additional source of exposure through the inhalation of $\mathrm{PCBs}$, as their sera contained elevated proportions of CB-28, CB-60, CB-66, CB-74, CB-105 and CB-118.

Whilst there were no clear trends in enantiomer signatures with the exposure type and duration identified in this study, there was a weak correlation between the EFs of CB-95 and CB-149 with EC7 PCB concentrations, suggesting that there may be a concentration dependent element to the metabolism of these congeners in humans. The extent of enantioselective metabolism or excretion in several workers was so great it resulted in the near complete removal the E2 enantiomer.

Supplementary data to this article can be found online at http://dx. doi.org/10.1016/j.envint.2015.04.006.

\section{Acknowledgements}

The authors would like to thank the following: Ann Tanderitispole and Chris Gallagher (University of Strathclyde) for their assistance with sample analysis and the Scottish Funding Council and EPSRC grant EP/D013739/2 for funding in association with the University of Strathclyde Laboratory, Corina Brimacombe and Terry Kolic for their help with the HRMS analysis of chiral PCBs, Alec Kettle (LECO) for his help and support with the research, and all the volunteers of this study who gave blood. Finally, David Megson would like to thank Plymouth University for funding this project as part of his $\mathrm{PhD}$ research.

\section{References}

Akins, J.R., Waldrep, K., Bernert, J.T., 1989. The estimation of total serum-lipids by a completely enzymatic summation method. Clin. Chim. Acta 184, 219-226.

Axelrad, D.A., Goodman, S., Woodruff, T.J., 2009. PCB body burdens in US women of childbearing age 2001-2002: an evaluation of alternate summary metrics of NHANES data. Environ. Res. 109, 368-378.

Brown, J.F., Lawton, R.W., 2001. Factors controlling the distribution and levels of PCBs after occupational exposure. In: Robertson, L.W., Hansen, L.G. (Eds.), PCBs Recent Advances in Environmental Toxicology and Health Effects. The University Press of Kentucky.

Chu, S., Covaci, A., Schepens, P., 2003. Levels and chiral signatures of persistent organochlorine pollutants in human tissues from Belgium. Environ. Res. 93, 167-176.

DeCaprio, A.P., Johnson, G.W., Tarbell, A.M., Carpenter, D.O., Chiarenzelli, J.R., Morse, G.S Santiago-Rivera, A.L., Schymura, M.J., Akwesasne Task Force E, 2005. Polychlorinated biphenyl (PCB) exposure assessment by multivariate statistical analysis of serum congener profiles in an adult Native American population. Environ. Res. 98, 284-302.

Focant, J.F., Sjodin, A., Patterson Jr., D.G., 2004. Improved separation of the 209 polychlorinated biphenyl congeners using comprehensive two-dimensional gas chromatography-time-of-flight mass spectrometry. J. Chromatogr. A 1040, 227-238.

Focant, J.F., Eppe, G., Massart, A.C., Scholl, G., Priard, C., De Pauw, E., 2006. Highthroughput biomonitoring of dioxins and polychlorinated biphenyls at the subpicogram level in human serum. J. Chromatogr. A 1130, 97-107.

Harju, M., Danielsson, C., Haglund, P., 2003. Comprehensive two-dimensional gas chromatography of the 209 polychlorinated biphenyls. J. Chromatogr. A 1019, 111-126.

Harner, T., Wiberg, K., Norstrom, R., 2000. Enantiomer fractions are preferred to enantiomer ratios for describing chiral signatures in environmental analysis. Environ. Sci. Technol. 34, 218-220.

Harrad, S.J., Hazrati, S., Ibarra, C., 2005. Concentrations of polychlorinated biphenyls in indoor air and polybrominated diphenyl ethers in indoor air and dust in Birmingham, United Kingdom: implications for human exposure. Environ. Sci. Technol. 40, 4633-4638.
Harrad, S., Ren, J.Z., Hazrati, S., 2006. Chiral signatures of PCB\#s 95 and 149 in indoor air, grass, duplicate diets and human faeces. Chemosphere 63, 1368-1376.

Helsel, D.R., 2006. Fabricating data: how substituting values for nondetects can ruin results, and what can be done about it. Chemosphere 65, 2434-2439.

Herrick, R.F., Meeker, J.D., Altshul, L., 2011. Serum PCB levels and congener profiles among teachers in PCB-containing schools: a pilot study. Environ. Heal. 10.

Jain, R.B., Wang, R.Y., 2011. Association of caffeine consumption and smoking status with the serum concentrations of polychlorinated biphenyls, dioxins, and furans in the general U.S. population: NHANES 2003-2004. J. Toxicol. Environ. Health, Part A 74, $1225-1239$

Jaspers, V.L.B., Megson, D., O'Sullivan, G., 2013. Chapter 7, POPs in the terrestrial environment. In: O'Sullivan, G., Sandau, C.D. (Eds.), Environmental Forensics for Persistent Organic Pollutants. Elsevier.

Johnson, G.W., Quensen III, J.F., Chiarenzelli, J.R., Coreen Hamilton, M., 2006. Polychlorinated Biphenyls. In: Morrison, R.D., Murphy, B.L. (Eds.), Environmental Forensics Contaminant Specific Guide. Academic Press.

Johnson, G.W., Ehrlich, R., Full, W., Ramos, S., 2007. Chapter 7: principal components analysis and receptor models in environmental forensics. In: Morrison, R., Murphy, B.L. (Eds.), An Introduction to Environmental Forensics, 2nd ed. Elsevier, Amsterdam, pp. 207-272.

Kodavanti, P.R.S., Curras-Collazo, M.C., 2010. Neuroendocrine actions of organohalogens: thyroid hormones, arginine vasopressin, and neuroplasticity. Front. Neuroendocrinol. 31, 479-496.

Kohler, M., Tremp, J., Zennegg, M., Seiler, C., Minder-Kohler, S., Beck, M., Leinemann, P., Wegmann, L., Schimi, P., 2005. Joint sealants: an overlooked diffuse source of polychlorinated biphenyls in buildings. Environ. Sci. Technol. 39, 1967-1973.

Korytar, P., Haglund, P., de Boer, J., Brinkman, U.A.T., 2006. Comprehensive twodimensional gas chromatography for the analysis of organohalogenated microcontaminants. TrAC Trends Anal. Chem. 25, 373-396.

Letcher, R.J., Klasson-Wehler, E., Bergman, Å., 1999. Methylsulfone and hydroxylated metabolites of polychlorinated biphenyls. In: Passivita, J. (Ed.), The Handbook of Environment Chemistry. Part K: New Types of Persistent Halogenated Compounds vol. 3. Springer-Verlag, Heidelberg, pp. 315-360.

Longnecker, M.P., 2001. Endocrine and other human health effects of environmental and dietary exposure to polychlorinated biphenyls. In: Robertson, L.W., Hansen, L.G. (Eds.), PCBs Recent Advances in Environmental Toxicology and Health Effects. The University Press of Kentucky.

Luotamo, M., Patterson Jr., D.G., Needham, L.L., Aitio, A., 1993. Concentrations of PCB congeners in sera from workers with past and present exposure. Chemosphere 27, 171-177.

Megson, D., O'Sullivan, G., Comber, S., Worsfold, P.J., Lohan, M.C., Edwards, M.R., Shields, W.J., Sandau, C.D., Patterson Jr., D.G., 2013a. Elucidating the structural properties that influence the persistence of PCBs in humans using the National Health and Nutrition Examination Survey (NHANES) dataset. Sci. Total Environ. 461-462, 99-107.

Megson, D., Kalin, R.B., Worsfold, P., Gauchotte-Lindsay, C., Patterson Jr., D.G., Lohan, M.C., Comber, S., Brown, T.A., O'Sullivan, G., 2013b. Fingerprinting polychlorinated biphenyls in environmental samples using comprehensive two-dimensional gas chromatography with time-of-flight mass spectrometry. J. Chromatogr. A 1318, $276-283$.

Oki, M., 1983. Recent advances in atropisomerism. Top. Stereochem. 14, 1-81.

O'Sullivan, G., Sandau, C.D., 2013. Environmental Forensics for Persistent Organic Pollutants. Elsiever.

Quinn, C.L., Wania, F., 2012. Understanding differences in the body burden-age relationships of bioaccumulating contaminants based on population cross sections versus individuals. Environ. Health Perspect. 120 (4), 554-559.

Robson, M., Harrad, S., 2004. Chiral PCB signatures in air and soil: implications for atmospheric source apportionment. Environ. Sci. Technol. 38, 1662-1666.

Schantz, S.L., Seo, B.W., Wong, P.W., Pessah, I.N., 1997. Long-term effects of developmental exposure to 2,2',3,5',6-pentachlorobiphenyl (PCB 95) on locomotor activity, spatial learning and memory and brain ryanodine binding. Neurotoxicology 18, 457-467.

Weintraub, M., Birnbaum, L.S., 2008. Catfish consumption as a contributor to elevated PCB levels in a non-Hispanic black subpopulation. Environ. Res. 107, 412-417.

Wong, C.S., Hoekstra, P.F., Karlsson, H., Backus, S.M., Mabury, S.A., Muir, D.C.G., 2002. Enantiomer fractions of chiral organochlorine pesticides and polychlorinated biphenyls in standard and certified reference materials. Chemosphere 49, 1339-1347.

Wu, X.A., Kammerer, A., Lehmler, H.J., 2014. Microsomal oxidation of 2,2',3,3',6,6'hexachlorobiphenyl (PCB 136) results in species-dependent chiral signatures of the hydroxylated metabolites. Environ. Sci. Technol. 48, 2436-2444.

Zapadlo, M., Krupcik, J., Kovalczuk, T., Majek, P., Spanik, I., Armstrong, D.W., Sandra, P., 2011. Enhanced comprehensive two-dimensional gas chromatographic resolution of polychlorinated biphenyls on a non-polar polysiloxane and an ionic liquid column series. J. Chromatogr. A 1218, 746-751. 